\title{
La locura y la psiquiatría en México: un balance historiográfico
}

Maria Cristina Sacristán INSTITUTO MORA

$\mathrm{E}$

n toda investigación histórica, los resultados finales dependenengran parte de la orientación, los métodos y las fuentes utilizadas, cuya elección estriba, por lo general, en la formación del investigador. En un intento quizá pretencioso por orientar el futuro quehacer de la historiografia mexicana sobre la locura y la psiquiatría, se analizarán de manera global los trabajos realizados hasta ahora, considerando el perfil de los autores, las temáticas, las regiones del país y los tiempos históricos privilegiados y los desdeñados, las características de las fuentes consultadas, la originalidad y alcance de las investigaciones y su difusión, para finalmente proponer algunas alternativas a la investigación futura.
En la bibliografia consultada no se estableció previamente un criterio de selección, procurando fuera lo más exhaustiva posible. Como podrá apreciarse, algunas investigaciones redundan sobre los mismos temas; pareció conveniente incluirlas precisamente porque reflejan una tendencia que, sin embargo, no ha dado buenos resultados, por lo que sería deseable evitarla.

Los títulos aquí analizados se agruparon temáticamente de la siguiente manera: 1) General, 2) Hospitales de locos y manicomios, 3) Patologías individuales, 4) Tratados de medicina y de psiquiatría, 5) Diagnóstico y terapéutica de la locura y 6) Centros de enseñanza e investigación de la psiquiatría.

\section{1}


1) General. Este rubro comprende historias de la medicina que incluyen capítulos dedicados a la psiquiatría e historias particulares de la locura que abarcan desde los tiempos prehispánicos hasta la actualidad, haciendo mayor hincapié en los aspectos institucionales (hospitales, enseñanza de la psiquiatría, centros de investigación) $\mathrm{y}$, en menor medida sobre el diagnóstico y la terapéutica.

2) Hospitales de locos y manicomios. Aquí incluí trabajos que rememoran el origen de la institución hospitalaria comenzando por destacar la biografia del fundador, los motivos que guiaron su empresa y los medios humanos -en ocasiones los mismos locos-y materiales de los que se valió para ałcanzar la construcción y sostenimiento de los hospitales. Abordan también la historia administrativa, los avatares presupuestales, las transformaciones habidas por motivos políticos totalmente ajenas a decisiones internas del hospital -sobre todo bajo los gobiernos del general Santa Anna-, el diseño arquitectónico, y las demoliciones y reconstrucciones que vivieron. Son minoría los estudios que contemplan la asistencia terapeútica de los locos en dichos hospitales, las patologías en que viven sumidos los insensatos, las altas y bajas, las características del personal del hospital, así como las condiciones generales de alimentación, vestido, higiene, etcétera.

3)Patologías individuales. Son estudios pormenorizados de sujetos considerados locos por sus contemporáneos o percibidos así hoy en día por la psiquiatría actual aun cuando en su momento hayan sido concebidos como endemoniados o místicos, líderes rebeldes, herejes o delincuentes. La base documental de estas investigaciones procede de archivos judiciales, lo que las ha enriquecido en cuanto a originalidad e interpreta- ción. Además, en los tribunales se manifiesta el comportamiento de la sociedad hacia los insensatos, el diagnóstico de la enfermedad que aqueja al reo por parte de un facultativo y se evidencia, en la segunda mitad del siglo XIX, que la familia, la medicina y el derecho, pelean entre sí por obtener el control final del demente. Cuando se ha contado con la trayectoria personal del loco, se ha interpretado su discurso, apoyándose en la teoría psicoanalítica.

4) Tratados de medicina y de psiquiatría. Como podría preverse, los estudios sobre el desarrollo de la psiquiatría a través de los textos utilizados para la enseñanza han sido coto casi privilegiado de los psiquiatras y los investigadores con formación médica. Estos compendios informan sobre la clasificación de las enfermedades mentales según su sintomatología y el remedio más apropiado para mermar su virulencia. Los historiadores de la psiquiatría han analizado tanto los testimonios españoles como los indígenas, advirtiéndose una auténtica fascinación por el Códice Badiano. De once estudios sobre la época prehispánica, seis son análisis exclusivamente de dicho documento, mientras los cinco restantes versan sobre el tratamiento de las enfermedades mentales fundamentalmente a través de esta obra. La consideración de que este testimonio es más confiable que los emanados de las plumas españolas sobre los tiempos prehispánicos, ha debido contribuir a que corriera tanta tinta. Además, los códices por sí mismos parecen tener un atractivo que les es consustancial.

5) Diagnóstico y terapéutica de las enfermedades mentales. Aunque la nosología de las enfermedades mentales es tratada en algunos trabajos clasificados en las divisiones anteriores, aquí es estudiada de manera exclusiva haciendo 
hincapié en la terapéutica. De nuevo los tiempos precolombinos y coloniales se llevan la mejor tajada a manos de médi$\cos$ y psiquiatras.

6) Centros de enseñanza e investigación de la psiquiatria. Los trabajos incluidos en esta sección son de acuñación reciente debido también a la juventud de la psiquiatría como disciplina universitaria y con un campo exclusivo de investigación. Es posible también que en nuestra revisión no estén bien representados, porque escapan a nuestro interés primordial.

Según un criterio más amplio, en esta evolución pudieran haber quedado comprendidas algunas monografias de historia social que tratan de forma colateral aspectos de la historia de la locura y la psiquiatría en México. Por ejemplo, el estudio ya clásico de Gonzalo Aguirre Beltrán, Medicina y magia. El proceso de aculturación en la estructura colonial incluye referencias al diagnóstico y terapéutica de la medicina española, india y negra. Los vencidos. Los indios del Peri frente a la conquista española (15301570) del historiador francés Nathan Wachtel expone, mediante fuentes indígenas mesoamericanas y andinas algunos comportamientos de los conquistadores españoles percibidos como "locura" por parte de la población sometida, pues escapaban a la lógica propia de la visión del mundo indígena. Igualmente la reciente obra de Solange Alberro sobre el Tribunal.del Santo Oficio, Inquisición $y$ sociedad en' México, 1571-1700, menciona los padecimientos nerviosos que la reclusión prolongada en las cárceles inquisitoriales provocaba en los reos. Trabajos de esta índole no fueron incluidos en nuestra revisión porque tratan el problema de la locura de manera secundaria al no ser obras específicas sobre la historia de la psiquiatría.
La mayoría de los estudios aquí analizados fueron publicados a partir de la década de los sesenta de nuestro siglo, acaso debido al impulso que conoció la psiquiatría en México y a la supresión del porfiriano manicomio de "La Castañeda" que inauguraba una nueva etapa con la creación de las granjas-hospitales.

La formación médica de gran parte de los investigadores ha determinado en forma decisiva no tanto la elección del periodo histórico estudiado como las temáticas preferidas y las fuentes consultadas. Preocupados por la historia hospitalaria, los diagnósticos y los tratados de medicina, han dejado de lado otros aspectos de la historia de la locura más atendidos por los historiadores. Como éstos llegaron más tardíamente a su encuentro con la locura y la psiquiatría, ocuparon nuevos campos de investigación prácticamente intocados por sus antecesores, los médicos y psiquiatras. Han buscado insertar la historia mexicana en un ámbito europeo o americano a fin de explicar su desarrollo; comprender más que describir por qué unas sociedades generaron ciertas formas de locura y qué significado tuvo para ellas; analizar los cambios sufridos por la historia de la locura y de la psiquiatría en el marco de la historia general de México para comprender por qué actuaron y pensaron de otro modo los hombres en el pasado. Más que desentrañar las patologías específicas de acuerdo a las descripciones de las fuentes, interesó conocer las motivaciones inconscientes de los locos; en vez de absolverlos de delitos que en su tiempo les imputaron, poner al descubierto qué pretendieron decir a sus contemporáneos y cómo vivieron su locura; qué significaba estar o pasar por loco.

La época preferida por los estudiosos ha sido con mucho la colonial, que repre-
1 
senta más de la mitad de la producción, sin contar la de carácter general que dedica la mayor parte de sus páginas a los tres siglos del dominio español. En segundo lugar de importancia sigue el periodo prehispánico, privilegiándose a la cultura nahua quizá por ser la más docu. mentada. El México independiente, con todo y el desarrollo que vivió la psiquiatría en Europa durante la centuria decimonónica ha sido prácticamente dejado en el olvido por los estudiosos mexicanos y extranjeros.

Lo propio puede afirmarse de los trabajos regionales. El centralismo ha dominado de manera avasalladora la producción existente; historias que aun teniendo como marco a la nación, confunden y entremezclan la historia nacional con la capitalina. Los trabajos acerca de las fundaciones hospitalarias versan, la mayoría, sobre la ciudad de México. La descentralización parece iniciarse en los estudios que tratan sobre la psiquiatría del siglo XX, acaso porque ésta se extiende a diversas regiones del país.

La mayor parte de las fuentes consultadas por los autores han sido secundarias o contemporáneas a los hechos estudiados pero ampliamente difundidas. El escaso acercamiento a los archivos ha redundado 'en la proliferación de investigaciones un tanto repetitivas. De hecho, las más originales y las de mayor alcance en cuanto al progreso de la historiografia se han basado en repositorios de documentos inéditos. Fuerza es reconocer la imperiosa necesidad de abordar nuevas fuentes si queremos evitar un estancamiento en la producción: la hemerografia jurídica y médica, la literatura, las crónicas, las obras de los viajeros y los diccionarios.

Los principales problemas de difusión a los que se enfrenta la producción existente obedece a las características de las publicaciones. Las dos terceras partes son artículos vaciados en revistas de medicina y psiquiatría esto es, en un medio muy especializado y de escasa difusión entre los historiadores, quienes también suelen dar a la luz sus materiales en publicaciones propias de su ámbito.

Para modificar la tendencia actual en los estudios sobre la historia de la locura y de la psiquiatría con miras a lograr una interpretación que trascienda el empirismo en que parece verse envuelta, sería necesario orientar los estudios hacia dos vertientes. Una, la historia asistencial y médica de los hospitales para locos que contemple el sostén económico del hospital, el personal médico y de servicio, el análisis de los reglamentos y de los expedientes clínicos de los enfermos, el tratamiento médico y las condiciones de los internos en cuanto a estado general de salud, bienestar, alimentación, rutina diaria y en general, sobre la vida interna del hospital; finalmente, es necesario conocer la división de los enfermos por áreas, no siempre coincidentes con las recomendadas por los tratados de medicina. Por otro lado, una historia social de la locura que dé cuenta de la relación entre locos y cuerdos a través del comportamiento de la sociedad con los enfermos, de la concepción de la locura desde el punto de vista institucional a partir de los tratados de medicina, de los diagnóstico médicos en los hospitales y en los procesos judiciales y de la legislación civil y canónica. También es posible conocer la sabiduría popular en torno a las enfermedades mentales, discernible en los mencionados archivos judiciales, las crónicas de la época y la literatura.

\section{BIBLIOGRAFÍA}

\section{GENERAL}

-Álvarez Amézquita, José et al., Historia de 
la salubridad y de la asistencia en México, 4 vols., Secretaría de Salubridad y Asistencia, México, 1960.

-Belsasso, Guido, "La psiquiatría en México. Desarrollo histórico", en Actualidades médicas, vol. III, núm. 1, octubre, 1971, pp. 114-119.

-Calderón Narváez, Guillermo, "La salud mental en México. Antecedentes históricos y enfoque actual", en Acta Psiquiátrica y Psicológica de América Latina, vol. XVI, núm. 3, 1970, pp. 234-239.

-Cooley, Eduardo, "Desarrollo institucional de la atención del psiquismọ en México", en Silvia Marcos (coord.), Manicomios y prisiones, Re-ediciones, México, 1983, pp. 57-62.

-De la Fuente, Ramón y Carlos Campillo, "La psiquiatría en México: una perspectiva histórica", en Gaceta Médica de México, vol. CXI, núm. 5, mayo de 1976, pp. 421-436.

-Flores y Troncoso, Francisco de Asís, Historia de la medicina en México desde la época de los indias hasta la presente, 4 vols., Instituto Mexicano del Seguro Social, México, 1982.

-León, A.C. y H. Roselli, Latin America in world bistory of psycbiatry, Bailliere Tindall, Londres, 1975.

-Ocaranza, Fernando, Historia de la medicina en México, Laboratorio Midy, México, 1934.

-Ramírez Moreno, Samuel, La asistencia psiquiátrica en México, Secretaría de Salubridad y Asistencia, México, 1950.

-Somolinos D'Ardois, Germàn, Historia de la psiquiatria en México, Secretaría de Educación Pública, México, 1976.

-Suárez Escobar, Marcela, "La locura y los hospitales para dementes en Nueva España", en Revista $A$, vol. VIU, núm. 22, septiembrediciembre 1987, pp. 121-137.

\section{HOSPITAIES DE locos Y MANICOMIOS}

-Berkstein Kanarek, Celia, "El Hospital del Divino Salvador", tesis de licenciatura, Facultad de Filosofía y Letras, UNAM, México, 1981.

-Calderón Narváez, Guillermo, "Hospitales psiquiátricos en México", en Revista Mexicana de Neurologia y Psiquiatria, núm. 7, 1966, pp. 111.
-Delgado Roig, Juan, Fundaciones psiquiátricas en Sevilla y nuevo mundo, $\mathrm{Paz}$ Montalvo, Madrid, 1948.

-Fernández del Castillo, Francisco, "San Hipólito en la historia y en la medicina de México", en El Médico, año VI, núm. 7, octubre de 1956, pp. 97-112.

"El hospital de San Hipólito en la historia general y en la historia de la medicina en México II", en El Médico, año vi, núm. 8, noviembre de 1956, pp. 86-102.

"Bernardino Álvarez, iniciador de la atención neuropsiquiátrica en México", en Gaceta Médica de México, México, vol. XCVI, núm. 9, septiembre de 1966, pp. 10131022.

"El manicomio general de La Castañeda", en $E l$ Médico, año IV, núm. 2, marzo de 1955, p. 58.

-Jiménez Olivares, Ernestina, "Bernardino Álvarez: su vida y su obra", en Prensa Médica Mexicana, año XXXV1, núms. 11-12, noviembre-diciembre de 1972, pp. 395-402.

-Martin, Cherly, The San Hipólito hospitals of colonial Mexico, 1566-1 704, tesis doctoral, Tulane University, Nueva Orleans, 1976.

-Muriel, Josefina, Hospitales de la Nueva España, 2 vols., Editorial Jus, México, 1956. "El modelo arquitectónico de los hospitales para dementes en la Nueva España", en Retablo barroco a la memoria de Francisco de la Maza, UNAM, México, 1974, pp. 115-125.

-Ramírez Moreno, Samuel, "Datos históricos sobre los manicomios y la psiquiatría en México", en Revista Mexicana de Psiquiatria, Neurologia y Medicina Legal, vol. I, mayo de 1934, pp. 7-19.

"El Dr. Rafael Lavista y la Quinta de Salud de Tlalpan", en Revista Mexicana de Psiquiatría, Neurologiay Medicina Legal, vol. II, núm. 71, 1935, pp. 25-28.

-Santiago Cruz, Francisco, Los hospitales de México y la caridad de Don Benito, Editorial Jus, México, 1959.

-Somolinos D'Ardois, Germán, "Comentario al trabajo 'Bernardino Álvarez, iniciador de la atención neuropsiquiátrica en México' ", en Gaceta Médica de México, vol. XIVI, 1966, pp. 1023-1025. 
-Viqueira, Carmen, "Los hospitales para 'locos e inocentes' en Hispanoamérica y sus antecedentes españoles", en Revista de Medicina y Ciencias Afines, vol. XxII, núm. 270, 1965, pp. 1-34.

\section{PATOLOGÍAS INDIVIDUALES}

-Fernández del Castillo, Francisco, "Un dictamen neuropsiquiátrico en 1775 ; contribución a la historia de la neuropsiquiatría en México", en El Médico, año vIII, núm. 10, enero, 1959, pp. 41-46.

-Gruzinski, Serge, "Delires et visions chez les indiens du Mexique", en Mélanges del'Ecole Française de Rome, vol. LXXXVI, 1974, pp.445480.

-Jiménez Olivares, Ernestina, "El proceso del doctor José Ignacio Brizuela", en Prensa Médica Mexicana, año. XxxvI, núms. 11-12, noviembre-diciembre de 1972, pp. 403-408.

"El proceso contra el doctor don Juan de la Peña", en Prensa Médica Mexicana, año XXXvil, núms. 11-12, noviembre-diciembre de 1972, pp. 409-411.

"Psiquiatría e Inquisición. Proceso contra el cirujano Juan Luis de Torres" en Doctor Francisco Fernández del Castillo. 50 años de vida profesional, UNAM, México, 1973, pp. 105-115.

"Psiquiatría e Inquisición. El proceso de fray Salvador de Victoria", en Revista de la Facultad de Medicina, UNAM, vol. XXVI, año XXVI, núm. 10, 1983, pp. 430-433. "Psiquiatría e Inquisición. El proceso de don Guillén de Lampart", en Semana Médica de México, vol. CL, año XXXI, núm. 11, 1, 333, 14 de septiembre de 1984, pp. 339-348.

-Sacristán, María Cristina, Locura e Inquisición en el virreinato de Nueva España (1571-1760), Fondo de Cultura Económica/ El Colegio de Michoacán, México, 1991 (en prensa).

"Pecadores inocentes: algunos avances sobre la locura en Nueva España, 1571-1760", en Seminario de Historia de las Mentalidades, Del dicho al becbo... Transgresiones y pautas culturales en Nueva España, Instituto Nacional de Antropología e Historia, México, 1989, pp. 29-42.
-Van Young, Eric, "Millenium on the northern marches: the mad mesiah of Durango and popular rebellion in Mexico, 1800-1815", en Comparative Studies in Society and History, vol. XXViII, núm. 3, julio de 1986, pp. 385-413. "Agustin Marroquin: the sociopath as rebel", en Judith Ewell y William $\mathrm{H}$. Beezley (comps.) The buman tradition in Latin America. The nineteenth century, A scholarly resources imprint, Wilmington, 1989, pp. 17-38.

\section{TRATADOS DE MEDICINA Y PSIQUIATRÍA}

-Calderón Narváez, Guillermo, "Conceptos psiquiátricos de la medicina azteca contenidos en el Códice Badiano escrito en el siglo XV", en Revista de la Facultad de Medicina de México, núm. 7, 1985, p. 229.

-De la Peña, Ignacio y Carlos Viesca Treviño, "El tratamiento de las enfermedades mentales en el Códice Badiano", en Estudios sobre etnobotánica y antropología médica II, 1977, pp. 21-26.

-Emmart, E.W., The Badianus manuscript, The Johns Hopkins Press, Baltimore, 1940.

-Fernández del Castillo, Francisco, "El tratado breve de medicina y de todas las enfermedades por Agustín Farfán", en El Médico, año vil, núm. 2, mayo, 1957, pp. 67 y 114. , "La verdadera medicina, cirugía y astrología por el doctor Barrios", en $\mathrm{El} \mathrm{Mé-}$ dico, año vil, núm. 4 , julio 1957, pp. 20-22.

-Somolinos D'Ardois, Germán, "El libellus de medicinalibus indorum herbis. Su significación", en Gaceta Médica de México, vol. XCrv, núm. 3, marzo de 1964, pp. 211-216. "El Códice de la Cruz-Badiano; estudio histórico", en Revista Estomatologia, vol. III, núm. 2, diciembre de 1965, pp. 3-8.

-Viesca, Carlos e Ignacio de la Peña, "Las enfermedades mentales en el Códice Badiano", en Estudios de Cultura Nábuatl, núm. 12, 1976, pp. 79-84.

\section{DIAGNÓSTICO Y TERAPÉUTICA DE LA LOCURA}

-Deutsch, A., The mentally ill in America: a bistory of their care and treatment from colonial times, Columbia University Press, Nueva York, 1952. 
-Díaz Infante, F., "Técnicas de psicoterapia practicadas por los mexicanos a principios del siglo XV", en A. Palacios, S. Ramírez y G. Valner (comps.), Psicoanálisis. La Técnica, Editorial Pax-México, México, 1963.

-Guerra, Francisco y H. Olivera, Las plantas fantásticas de México; introducción a la farmacología del teonanácatl, maribuana, canela, chicalote, adormidera, colorin, loco, peyote, cinicuiche, cozticzapotl, ololiubqui, camotillo, toloache, coca, banisteria y cobombrillo, Imprénta del Diario Español, México, 1954.

-Jiménez Olivares, Ernestina, "La interpretación de los sueños en el México prehispánico", en Psiquiatría. Órganooficial de la Asociación Psiquiátrica Mexicana, A.C., vol. viII, núm. 2, mayo-agosto de 1978, pp. 3032.

-Palacios L., Agustín, "La evolución del pensamiento de las ciencias psiquiátricas", en Neurología, Neurocirugia, Psiquiatria, vol. XXVII, número especial de aniversario, 1987, pp. 43-48.

- Vasconcelos, Daniel, "La enfermedad sagrada. Aspectos históricos y situación actual", en Gaceta Médica de México, vol. cXvII, núm. 1, enero de 1982, pp. 35-37.
-Velasco Alzaga, Jorge M., "Actividades sociales y legales ante el enfermo mental. Evolución histórica", en Psiquiatria. Organo oficial de la Asociación Psiquiátrica Mexicana, A.C., vol. I, septiembre-diciembre de 1968, pp. 15-19.

-Viesca, Carlos, "La enfermedad mental en el México colonial", en Psiquiatria. Organo Oficial de la Asociación Psiquiátrica Mexicana, A.C., vol. vi, núm. 3, septiembre-diciembre de 1976 , pp. 36-43.

"Los psicotrópicos y la medicina de los gobernantes entre los aztecas", en Estudios sobre etnobotánica y antropologia médica II, 1977, pp. 121-136.

\section{CENTROS DE ENSEÑANZA E} INVESTIGACIÓN DE LA PSIQUIATRÍA

-López, Manuel Isaías, "Historia de la enseñanza de la psiquiatría infantil en México", en Salud Mental, vol. vin, núm. 2, junio de 1985, pp. 17-19.

-Millán, Alfonso, "El desarrollo de la sociedad psicoanalítica mexicana y del Instituto Mexicano de Psicoanálisis", en Revista de Psicoanálisis, Psiquiatria y Psicologia, núm. 1, septiembre-diciembre de 1965, pp. 5-9. 


\section{SECUENCIA}

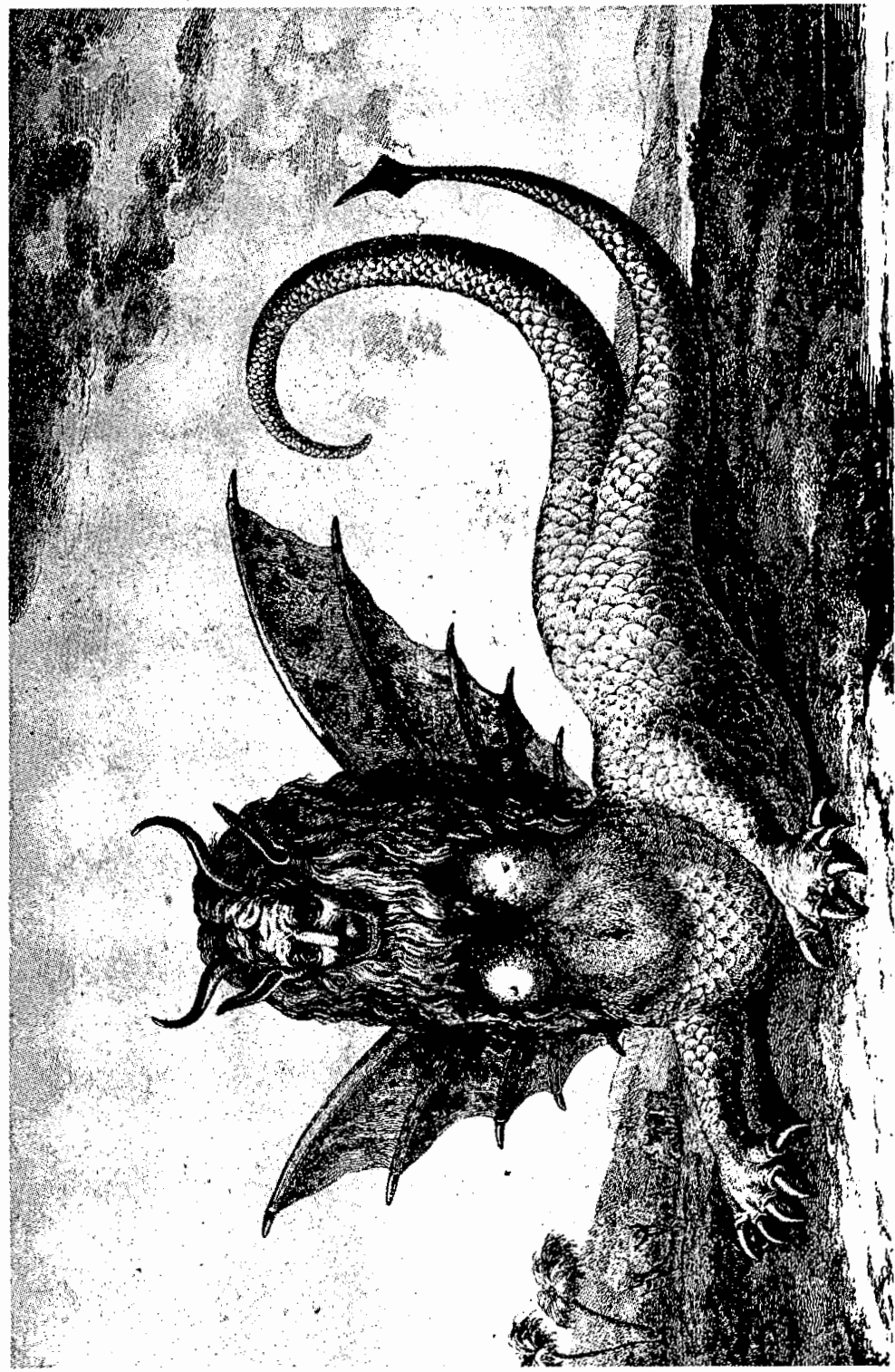

